\title{
The Effect of Different Solvents for Chitosan Solubilization on The Crystal Growth of in situ Prepared Hydroxyapatite
}

\author{
Mai K. Hassan, T. A. Elkhooly" and F. M. Reicha* \\ Biological Advanced Materials, Physics Department, Faculty of Science, \\ Mansoura University, Mansoura and \#Department of Ceramics, Inorganic \\ Chemical Industries Division, National Research Centre, Dokki, 12622, Cairo, \\ Egypt
}

\begin{abstract}
HITOSAN/hydroxyapatite (CS/HA) nanocomposites have been widely fabricated for potential use in bone defects due to their high biocompatibility and similarity to the natural bone. In the current study, HA nanocrystals were prepared by co-precipitation method in CS which was initially dissolved in different concentrations of acrylic acid and acetic acid (2 and $4 \%$ ). The effect of chitosan solvents on the morphology of HA nanocrystals, the crystallite sizes, and the physical binding with CS were investigated by different techniques including Fourier transform infrared spectroscopy (FT-IR), X-ray diffraction (XRD), X-ray photoelectron spectroscopy (XPS) and transmission electron microscope (TEM). The FT-IR and XPS results showed that the interaction between CS and HA in the nanocomposites is predominantly dependent on the type of solvent used to dissolve CS. When acetic acid was used, the interaction between CS and HA was stronger when compared to acrylic acid. Quantitative XPS results showed that the atomic percentage of nitrogen $(\mathrm{N} 1 \mathrm{~s})$ of the amine groups of CS was less in the case of the composites prepared after dissolving CS in acrylic acid compared to acetic acid. The amine groups of CS $\left(-\mathrm{NH}_{2}\right)$ can start the nucleation of HA crystals and remain bounded to the surface of the grown crystal through chelation with calcium ions on the crystal facets. In both organic solvents, HA crystals were found to grow along their c-axis direction into rodshape but the growth in the width was more restricted in case of acetic acid. The results in the current study indicated that the type of CS solvents is determining the strength of the physical interaction between the CS and HA. This might be a crucial factor to control and enhance the mechanical and the biological behaviors of the nanocomposites.
\end{abstract}

Keywords: Chitosan, Hydroxyapatite, Acrylic acid, Acetic acid, Nanocomposite.

\section{Introduction}

Human confronts many problems caused by accidents, diseases, and aging during their lifetime. In the past, surgeons had very limited tools to solve these medical problems and to replace the damaged tissues of human body [1]. For instance, the defects in bones have been considered as the most difficult problem in maxillofacial and oral surgery [2]. The methods of grafting as allograft, autograft and other therapies are not enough to solve all the problems of bones and also have some significant side effects [3]. New approach for repairing bone has been developed by using the concept of tissue engineering which is based on the fabrication of various three-dimensions (3D) scaffolds as carriers for osteogenic cells [4].
In bone tissue engineering, versatile biomaterials are used which are different in their origin from naturally occurring biopolymers [5].

One of these synthetic materials is hydroxyapatite $\left(\mathrm{Ca}_{10}\left(\mathrm{PO}_{4}\right)_{6}(\mathrm{OH})_{2}\right.$; $\left.\mathrm{HA}\right)$ which has been used as an inorganic materials that is similar to the minerals in human bone. It is the most stable crystalline form of calcium phosphates in the aqueous solution and it is highly biocompatible, osteoconductive, bioactive and non-toxic to human cells [6]. However, the use of HA in powder form has many problems for example it is not easy to handle during surgery and also it has poor mechanical properties. Therefore, preparing $\mathrm{HA}$ in polymers such as $\mathrm{CS}$ is an alternative approach to solve this problem. 
Chitosan is a linear polysaccharide which has many interesting properties in the field of regenerative medicine such as anti-bacterial effect [7]. The physicochemical properties of HA nanocrystals prepared in organic polymers is highly dependent on the experimental condition such as $\mathrm{pH}$ value and subsequently on the degree of ionization of functional groups of the polymers [8]. Because of the biocompatibility, biodegradability and high mechanical strength of this composite, it was used as tissue scaffold, and in reinforcing applications [9]. Generally, there is several ways to produce such composite materials either by mixing the solid inorganic powder with organic polymer or by preparing in situ preparing the inorganic materials from their ionic precursors in the polymer. The most used method is the in situ co-precipitation synthesis [10].

In situ preparation of CS/HA nanocomposites is beneficial because of the interaction between $\mathrm{CS}$ and the ionic precursors of HA. Usually $\mathrm{CS}$ is dissolved in weak acids then mixed with calcium $\mathrm{Ca}^{2+}$ or phosphate $\mathrm{PO}_{4}^{3-}$ ions thereafter, the self-assembly of these ions occur as a result of electrostatic interaction with the functional groups of the polymer, e.g. COO- $\mathrm{vs} \mathrm{Ca}^{2+}$ or $\mathrm{PO}_{4}^{3-}$ vs $\mathrm{NH}_{3}^{+}$[11]. Additionally, HA crystals carry two different protein binding sites on its surface, e.g. $\mathrm{P}$ and $\mathrm{C}$ sites that can bind to positively cationic groups such as $\mathrm{NH}_{3}^{+}$or negatively charged anionic groups such as $\mathrm{COO}^{-}$, respectively [12].

Acrylic acid (AA) is widely used in the formation of several functional materials, such as adhesives and textile treatment agents and water absorbent polymers [13]. Acrylic and acetic acids can be used to dissolve CS by donation of protons to CS amine groups [14]. Many previous works suggested that the particle size of HA can be controlled by adjusting the concentration of organic acids [15]. Yamaguchi et al. [16] investigated the effect of CS dissolved in variety of weak acids including citric, acetic, lactic, and malic acid on HA formation. The results showed that organic acids which contain more than two carboxyl groups, leads to the formation of amorphous HA, however, monocarboxylic acids such as acetic acid and lactic acid leads to the growth of different morphologies of crystalline HA.

The interaction between HA and polymer matrices might be dependent on the type of organic solvent of the polymer. Therefore, the main target of the current study is to explore the effect of various organic weak acids such as acetic and acrylic acids on the interaction between CS and HA. Furthermore, the change in morphology of HA nanoparticles and also the ability of these acids to modify the surface of HA will be also considered.

\section{Experimental}

\section{Materials}

Chitosan powders $\mathrm{Mw}=600,000-800,000$, degree of deacetylation $>85 \%$ was supplied by Acros Organics, USA , Acrylic acid AA (99\% Aldrich), Calcium hydroxide $\mathrm{Ca}(\mathrm{OH})_{2}$ (ANALAR,UK), Orthophoric acid $\mathrm{H}_{3} \mathrm{PO}_{4}(98 \%)$ was provided by BDH chemicals LTD Poole, England); sodium hydroxide $(\mathrm{NaOH})$. All the chemicals were used as received without any purification.

\section{Preparation of hydroxyapatite nanoparticles}

Hydroxyapatite nanoparticles were prepared using co-precipitation method, $5.3 \mathrm{gm}$ of $\mathrm{Ca}(\mathrm{OH})_{2}$ were first dissolved in $50 \mathrm{ml}$ deionized water at $\mathrm{pH}=12.35$ and then $1.89 \mathrm{ml}$ of $\mathrm{H}_{3} \mathrm{PO}_{4}$ was added to $50 \mathrm{ml}$ of deionized water then dropped into the above solution with magnetic stirring. After addition of $\mathrm{H}_{3} \mathrm{PO}_{4}, 5 \mathrm{M} \mathrm{NaOH}$ was added to adjust the reaction mixture to $\mathrm{pH}=9$. The final resulting slurries were aged for $48 \mathrm{hr}$ at room temperature before filtered and washed several times with deionized water then finally dried in vacuum oven at $50^{\circ} \mathrm{C}$.

\section{Preparation of CS/HA nanocomposites}

CS/HA composites were prepared by the chemical precipitation methods similar to the work published by Li et al. [17]. CS aqueous solution with concentration of $1.5 \mathrm{wt} \%$ was prepared by dissolving CS in different percentages of acrylic acid (AA) and acetic acid (Ac); 2 and $4 \%$ with overnight stirring to get a perfectly transparent solution. Thereafter, the CS solution was mixed with $10 \mathrm{wt} \%$ solution of $\mathrm{H}_{3} \mathrm{PO}_{4}$. The starting content of these reagents were scaled according to the final $\mathrm{CS} / \mathrm{HA}$ weight ratios of $30 / 70 \mathrm{wt} \%$. Meanwhile, the mixed solutions were then dropped slowly into $\mathrm{Ca}(\mathrm{OH})_{2}$ dissolved in deionized water, the dropping speed was adjusted about $3 \mathrm{ml} \mathrm{min}{ }^{1-}$ and the $\mathrm{pH}$ of resulting slurry was adjusted at $\mathrm{pH}=9$ with $5 \mathrm{M} \mathrm{NaOH}$ before aging for $24 \mathrm{hr}$. Finally, the resulting slurry was filtered, and washed with deionized water several times then dried in vacuum oven at $50^{\circ} \mathrm{C}$.

\section{Characterization}

Fourier transform infrared spectroscopy (FTIR) analysis was used to investigate the functional 
groups of polymer and HA. Before analysis, 2.5$3.0 \mathrm{mg}$ of nanocomposites powders were mixed with $300 \mathrm{mg} \mathrm{KBr}$ in the mortar and grinded into fine powder then pressed into solid disks. FTIR spectra were recorded using Mattson 5000 FTIR spectrophotometer at $25^{\circ} \mathrm{C}$ in the range of 400 to $4000 \mathrm{~cm}^{-1}$, resolution $4 \mathrm{~cm}^{-1}$.

X-Ray Photoelectron Spectroscopy (XPS) was used to analyze the surface chemistry of a material, the elemental composition, electronic state and chemical state of the elements within CS/HA nanocomposites. XPS survey and highresolution spectra of all grinded powders were collected using X-ray photoelectron spectroscopy (XPS, ESCALAB 250Xi, Thermo Scientific) equipped with a monochromatic $\mathrm{Al} \mathrm{K} \alpha \mathrm{X}$-ray source operating at $15 \mathrm{kV}, 10 \mathrm{~mA}$. Survey spectra were collected using an average of one scan with pass energy of $100 \mathrm{eV}$ and energy step size of $1 \mathrm{eV}$ running from 1400 to $0 \mathrm{eV}$. High-resolution spectra of elements such as O1s, N1s, C1s, Ca2p, and P2p were measured using an average of 3 scans with pass energy $30 \mathrm{eV}$ and a step size of $0.05 \mathrm{eV}$.

The incident beam was focused on a spot with a diameter of $500 \mu \mathrm{m}$ and the acquisition time was varied between 1 minute and 2 minutes for XPS survey and high-resolution spectra, respectively. X-ray source and the obtained XPS spectra were calibrated against the binding energy of $\mathrm{C} 1 \mathrm{~s}$ in the aliphatic carbon $\left(\mathrm{CH}_{2}\right)$ at $284.8 \mathrm{eV}$. The high-resolution spectra were resolved into individual Gaussian-Lorentzian peaks using a least squares fitting PeakFIT program. The height and width of each peak was manually adjusted to provide a reasonable starting point for the curve fitting program. The coefficient of determination $\left(\mathrm{r}^{2}\right)$

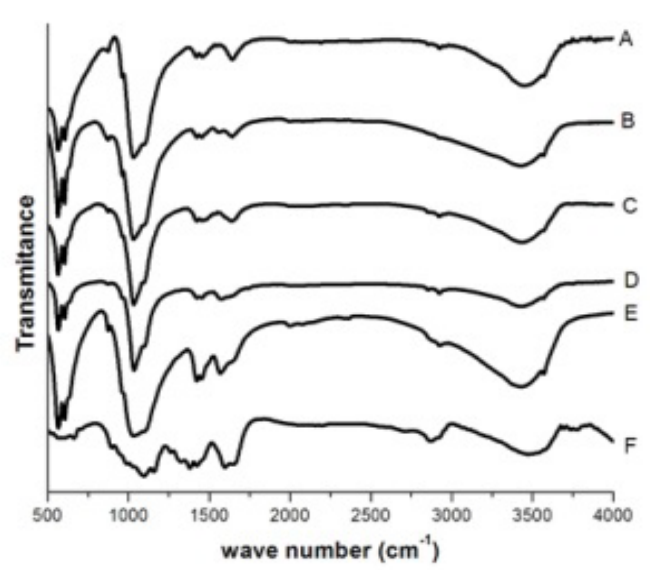

was $>0.999$ for all spectra.

X-ray Diffraction (XRD) patterns of pure $\mathrm{HA}$ and CS/HA powder were collected by using Rigaku X-ray diffractometer. The monochromatic X-rays with a wavelength of $0.1542 \mathrm{~nm}$ were generated using a $\mathrm{Cu} \mathrm{K} \alpha$ source with an emission current of $200 \mathrm{~mA}$ and a voltage of $40 \mathrm{kV}$. Scans The samples were measured from 5 to 60 degree at a scan speed 4 degree $/ \mathrm{min}$.

Transmission Electron Microscopy (TEM; JEOL JEM -2100) was used to monitor the particle size of n-HA powder and HA/CS nanocomposites with an accelerating voltage of $200 \mathrm{kV}$.

\section{Results and Discussion}

$\mathrm{CS}$ is commonly dissolved in acetic acid however; many other solvents have been also reported such as formic, lactic and acrylic acids [18]. All these weak acids provide sufficient protons to the primary amine groups of CS which leads to their ionization and consequently solubilization of CS.

Fourier transform infrared spectroscopy (FT-IR)

Figure 1 represents FT-IR spectra of HA, CS and CS/HA composites prepared in CS which was dissolved in 2 and $4 \%$ acrylic and acetic acids. The pure nano-HA characteristic bands were detected at 1032, 962, 604, 565 and $473 \mathrm{~cm}^{-1}$ which were attributed to the stretching and bending vibrations of phosphate groups in HA structure. The characteristic bands at 565 and $604 \mathrm{~cm}^{-1}$ were related to $v_{4} \mathrm{O}-\mathrm{P}-\mathrm{O}$ bending, and bands at $962 \mathrm{~cm}^{-1}$ and $1032 \mathrm{~cm}^{-1}$ were assigned to, $v_{1}$ symmetric PO stretching, and $v_{3}$ asymmetric PO stretching [19]. Additionally, the

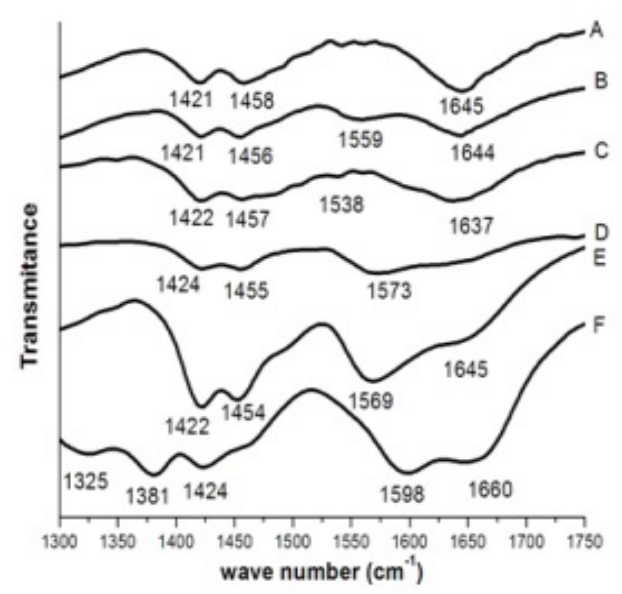

Fig. 1. FTIR spectra of HA/CS composite as a function of acrylic acids acid concentration.

where, A, B, C, D, E and F represent pure HA, HA/2AA/CS, HA/4AA/CS, HA/4Ac/CS, HA/2Ac/CS and pure CS, respectively. 
absorption bands at 1421 and $1458 \mathrm{~cm}^{-1}$ were assigned to carbonate ions $\mathrm{CO}_{3}{ }^{2-}$ and the one located at 3568 $\mathrm{cm}^{-1}$ was due to stretching vibration of structural hydroxyl groups of HA [20]. The characteristic peaks of Pure CS (Fig. 1 F) detected at 1647 and $1381 \mathrm{~cm}^{-1}$ were attributed to amide I and amide III, respectively. Furthermore, the band at $1598 \mathrm{~cm}^{-1}$ in CS was related to $\mathrm{N}-\mathrm{H}$ amide I [21] and this band in CS was shifted to lower wave number in all nanocomposites as shown in Fig.1 (B, C, D and E) due to the interaction of CS with HA [22]. This shift was previously attributed to the binding of chemical function groups of $\mathrm{CS}\left(-\mathrm{NH}_{2}\right.$ and $\left.-\mathrm{NHCO}^{-}\right)$and $\mathrm{PO}_{4}^{3-}$ via electrostatic interaction or $\mathrm{Ca}^{2+}$ via coordination bond [23]. Finally in the case of acrylic acid, the band at $1598 \mathrm{~cm}^{-1}$ suffered smaller shift which indicated that the in situ formed HA did not have strong interaction with CS's amino groups.

\section{$X$-ray photoelectron spectroscopy (XPS)}

XPS is commonly used to determine the chemical composition of about $10 \mathrm{~nm}$ depth of a solid surface. Therefore, in order to study the presence of CS on the surface of in situ prepared HA nanocrystals, high resolution XPS spectra of carbon (C1s) and nitrogen (N1s) were recorded. Figure 2 demonstrates high resolution spectra of $\mathrm{C} 1 \mathrm{~s}$ of pure HA without polymer addition while, Figure 3 represents the same element for CS/HA nanocomposites. Three kinds of peaks can be noticed in pure HA spectrum which were attributed to the adventitious organic contaminants or the adsorption of impurity hydrocarbons during the synthesis procedure. These peaks do not affect the interpretation of our results after CS addition [19, 24]. The peak located at $284.6 \mathrm{eV}$ was assigned to the $\mathrm{C}-\mathrm{C}$ and $\mathrm{C}-\mathrm{H}$ appeared in all samples and remained almost constant in their areas [24]. However, in all nanocomposite samples, new peak was observed in the range of 286.3 to $288 \mathrm{eV}$ which was related to the chemical bond between carbon and nitrogen or oxygen $[\mathrm{C}-(\mathrm{N}, \mathrm{O})]$. These bonds are directly related to amine $\left(\mathrm{NH}_{2}\right)$ or acetyl $\left(\mathrm{CH}_{3} \mathrm{COR}\right)$ groups in $\mathrm{CS}$ chains. The intensity and percentage area of this peak were increased in the case of acetic acid as shown in Fig. $3 \mathrm{C}$ and $\mathrm{D}$ but decreased in case of acrylic acid (Fig. 3 A and B). This indicates that the large amount of CS was loaded on the surface of HA when acetic acid was used as a solvent for CS. The peak at high binding energy in the range of 288.3 to $289.4 \mathrm{eV}$ are related to the carboxylic acid groups of AA and Ac acids which showed larger area in the case of acetic acid, probably due to the strong association between the protonated amine groups of CS and the ionized carboxylate of acrylic acid. Finally, new small spectral component at low binding energy $282.5 \mathrm{eV}$ is assigned to some carbon with a differential charging effect $\left(\mathrm{C}^{*}\right)$ observed in only acetic acid samples, this phenomenon is frequently observed in the ceramics $[19,25]$.

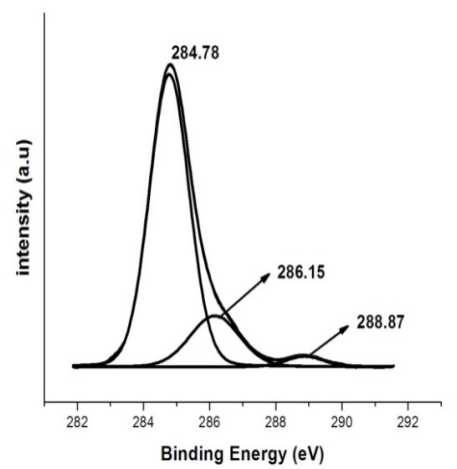

Fig. 2. High resolution C1s XPS spectra of pure HA.
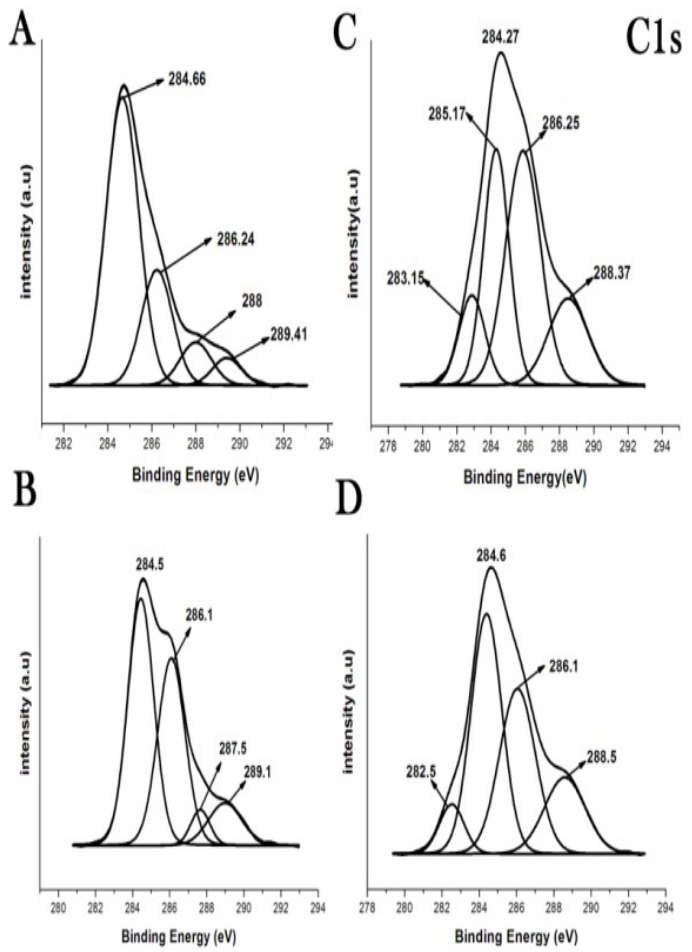

Fig. 3. High resolution C1s XPS spectra of HA/CS composite as a function of different acids content while (A) HA/CS/2AA (B) HA/ CS/4AA, (C) HA/CS/2Ac, (D) HA/CS/4Ac. 
On the other hand, Fig. 4 represents N1s spectrum which originates from amine or amide groups of CS chains [26]. In Fig. 4 (C, D) the peaks at 399.73 and 399.97 ev were assigned to the free amine group $\left(-\mathrm{NH}_{2}\right)$ which indicated the existence of CS on HA surface. The intensity and the atomic concentration of these peaks increased after dissolving CS in acetic acid (Table 1) indicating that CS was loaded more on the surface of HA [24]. Since the peaks at 399.23 and 399.5 ev are related to the non-protonated amine and the peaks at $401.3 \mathrm{ev}$ is assigned to protonated amine $\left(\mathrm{NH}_{3}^{+}\right)$. The later peak appeared only in the case of acetic acid and disappeared in the case of acrylic acid confirming the strong interaction between acrylic acid and HA surface that impaired the immobilization of CS on the surface of HA.

It has been reported by Shen et al. [27] that both charged $-\mathrm{COO}^{-}$and $-\mathrm{NH}_{3}^{+}$groups are the major functional groups of amino acid in protein sequence that facilitate the interaction with HA surface. Hence, in the case of acrylic acid, the strong interaction between its carboxylate group and HA surface might be responsible for blocking the adsorption sites of the amine groups of CS. But because the acetic acid is weaker acid than acrylic acid, CS was found to interact more on the surface of HA. The intensity and area of $-\mathrm{COO}^{-}$and $-\mathrm{NH}_{3}{ }^{+}$groups in the XPS spectra of all nanocomposite samples is proportional to the interaction energy with HA surfaces [27].

From FT-IR and XPS results, we showed that there are multiple interactions between polymers (CS) and HA crystals in CS/HA nanocomposites. Generally, there are different interactions between $\mathrm{Ca}^{2+}, \mathrm{PO}_{4}{ }^{3-}$ and $\mathrm{COO}^{-},-\mathrm{NH}_{2}$ which play important roles in the formation process of HA crystal. We proved that when acetic acid was used as CS solvent, more CS was loaded on the surface of HA. But in case of acrylic acid, the amount of CS was reduced on the surface of HA.

\section{$X$-ray diffraction $(X R D)$}

Figure 5 shows the XRD patterns of the pure HA and CS/HA nanocomposites. XRD diffraction showed that there are no significant differences in all samples and verified the formation of the hexagonal structure of HA crystals (JCPDS Card No. 9-0432) and no impurity or peaks of other calcium phosphate phases were detected [28]. There are two groups of XRD reflection peaks can be used to detect the HA formation one at $2 \Theta=25.9^{\circ}$ and the other group at $2 \Theta=32.9^{\circ}$ which corresponds to the 002 and 300 reflection peak which related to the (c) and (a) crystallographic axes, respectively. The mean crystal size $\left(\mathrm{D}_{(\mathrm{hkl})}\right)$ of these two reflections was calculated from the following Scherrer's equation and listed in Table 1:

$$
\left.D_{h k l}=0.9 \lambda\left(\operatorname{Cos} \theta_{h k l}\right) \beta_{h k l}\right)
$$

where $\beta_{\text {hkl }}$ is the full width at half maximum in radians, $\Theta$ is the diffraction angle for plane (hkl), $\mathrm{K}$ is the constant depending on crystal habit (chosen as 0.94) and $\lambda$ is the wavelength of $\mathrm{Cu} \mathrm{K \alpha}$ radiation $(1.5406 \AA)$ [12].

The crystallite sizes of both reflections (002) and (300) in (c) and (a) axes directions of the pure HA were recorded to be 46.8 and $25.8 \mathrm{~nm}$, respectively. The crystallite sizes of all nanocomposites were found smaller (Table 1) in the case of in situ formed $\mathrm{HA}$ in CS which is probably due to the interaction between the polymer and ceramics which lead to inhibition of its growth. However, the crystallite sizes and intensity of peaks increased with increasing the concentration of $\mathrm{AA}$, this because competitive adsorption of acrylic acid and CS on the surface of HA crystal which weaken the interaction of CS with HA leading to larger crystallite sizes. Rusu et al, [29] showed that the average crystallite size of HA nanoparticles can be controlled by varying the $\mathrm{pH}$ and/or the weight proportions of CS to inorganic ions concentration. In the case of HA/ gelatine nanocomposites reported by Ghang et al. [22], lower concentration of gelatine contributed to the development of bigger crystallites but higher concentration of gelatine resulted in formation of tiny crystallites of HA.
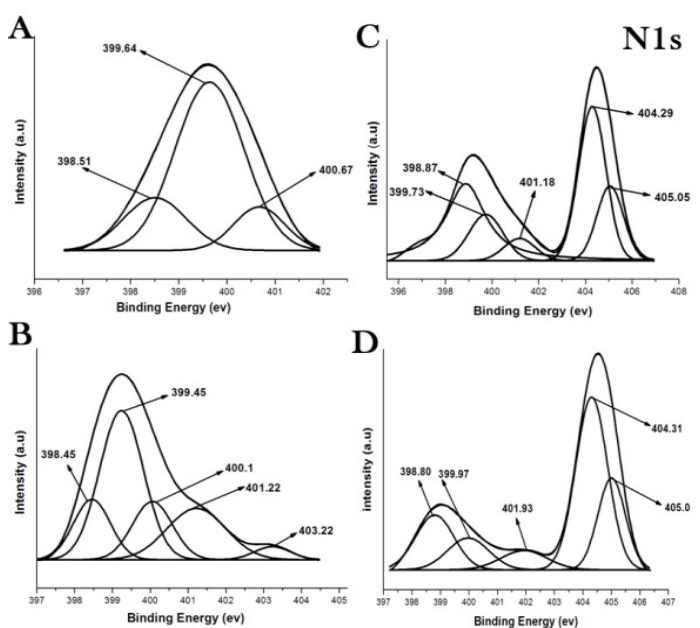

Fig. 4. High resolution N1s XPS spectra of the HA/ CS composite as a function of different acids content while (A) HA/CS/2AA (B) HA/CS/4AA, (C) HA/CS/2Aa and (D) HA/ CS/4Aa. 

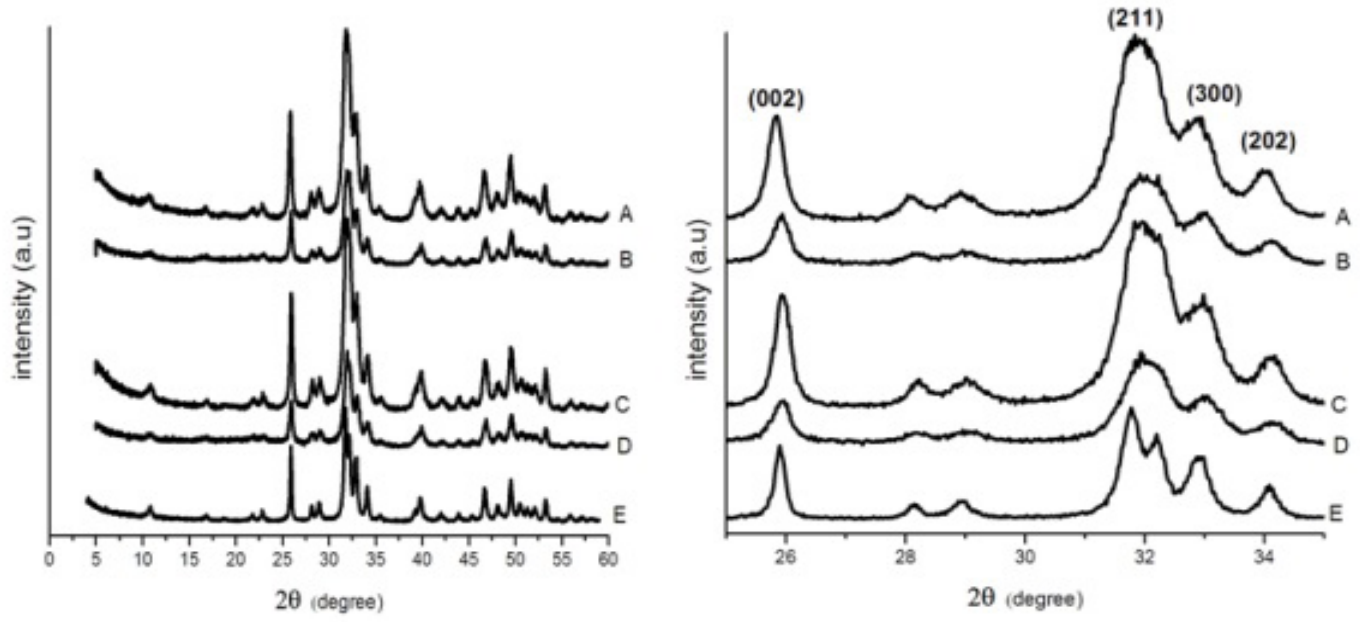

Fig. 5. XRD pattern of the HA/CS nanocomposites as a function of different acids concentration, (A) HA/CS/4AA, (B) HA/CS/4Ac (C) HA/CS/2AA (D) HA/CS/2Ac (E) Pure HA

TABLE 1. The atomic percentage of nitrogen at different concentrations of acetic acid and acrylic acids, crystallite sizes in two crystallographic planes of HA nanocrystal; hkl 002 and 300, and the dimension of the particles calculated from TEM images.

\begin{tabular}{|c|c|c|c|c|c|c|}
\hline Samples & $\begin{array}{c}\text { Atomic\% } \\
\text { N1s }\end{array}$ & $\begin{array}{c}\text { Crystal } \\
\text { size(300) D } \\
(\text { (nm) }\end{array}$ & $\begin{array}{c}\text { Crystal } \\
\text { size }(002) D \\
(\mathrm{~nm})\end{array}$ & $\begin{array}{l}\text { Particle } \\
\text { Length } \\
(\mathbf{n m})\end{array}$ & $\begin{array}{l}\text { Particle } \\
\text { Width } \\
(\mathbf{n m})\end{array}$ & Aspect ratio \\
\hline Pure HA & 0.29 & 25.8 & 46.8 & 77.7 & 18 & 4.3 \\
\hline $\mathrm{HA} / \mathrm{CS} / \mathbf{2 A A}$ & 0.54 & 17.6 & 28.1 & 49 & 18.9 & 2.5 \\
\hline $\mathrm{HA} / \mathrm{CS} / \mathbf{4 A A}$ & 0.81 & 16.4 & 27.8 & 45.3 & 22 & 2.1 \\
\hline $\mathrm{HA} / \mathrm{CS} / 2 \mathrm{Ac}$ & 1.29 & 15.3 & 27.2 & 43 & 17 & 2.6 \\
\hline $\mathrm{HA} / \mathrm{CS} / 4 \mathrm{Ac}$ & 1.18 & 15.8 & 23.3 & 40 & 14.1 & 2.8 \\
\hline
\end{tabular}

\section{Transmission Electron Microscopy (TEM)}

The morphology of pure HA and CS/HA nanocomposites synthesized with different ratios of acid is shown in Fig. 6 and 7, respectively. All samples displayed uniform rod-like structures with different aspect ratios (i.e., length/width) indicating that apatite crystals grow parallel to c crystallographic axes [30]. As presented in Table 1, the average particle size and the aspect ratios (i.e., length/width) were increased with increasing the concentration of acetic acids. The particle width was found smaller when acetic acid was used as a solvent for CS indicating that the enhanced interaction of CS with grown HA nanocrystals inhibit the growth in a-axis direction which decrease the particle width. On the other hand, by using acrylic acid as solvent, less CS was interacted with HA facets leading to bigger particle width. Liou et al. [31] suggested that in situ formed HA nanocrystals was synthesized in the presence of high molecular weight of polyacrylic acid (PAA). However, the dimension of these needlelike HA was found to be dependent on the $\mathrm{pH}$ value and concentration of the PAA. At low $\mathrm{pH}$, crystal growth of HA was inhibited in c-axis direction leading to smaller length. However, in the current study, this phenomena of inhibition of the growth of HA particle in c-axis direction was found negligible in the case of using acrylic acid monomer as compared to the effect of acrylic acid polymer which was reported in a previous work.

Besides, Fig. 7 (c, d, g, h) exhibited the selected area electron diffraction SAED which corresponding to different planes such as (002) planes for hexagonal HA [32]. This indicates that pure HA and HA in the nanocomposites grows 
along the c-axis direction into uniform rod like shape. Moreover, three planes of HA crystal (112, 211,300 ) were found overlapped and observed as a series of discrete strong spots in all samples. But in Fig. 7-h these planes appeared as a very sharp line of the electron pattern this indicates the formation of very tiny nanocrystals. While, in Fig. 8 we can see that the lattice space were measured to be around 2.631, 2.262 and 2.296 $\AA$ which were corresponded to the inter-planar distance of (202), (310), (212), respectively. These interplanar distances are related to some planes in HA crystal in consistent with XRD diffraction patterns.

\section{Conclusion}

Since the interaction between $\mathrm{CS}$ and $\mathrm{HA}$ is paramount importance in determining the quality of the resulting nanocomposites regarding their physical and biological properties for biomedical application. The current study focused on studying the effect of the type of CS solvent especially acetic and acrylic acids on the

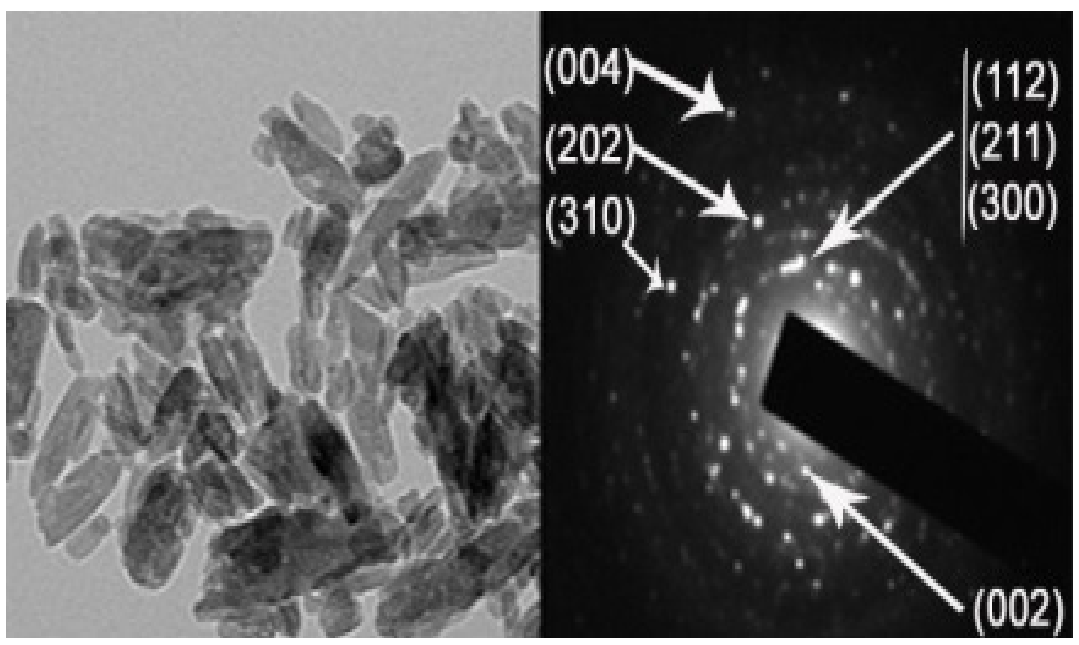

Fig. 6 TEM image and diffraction pattern of pure HA.
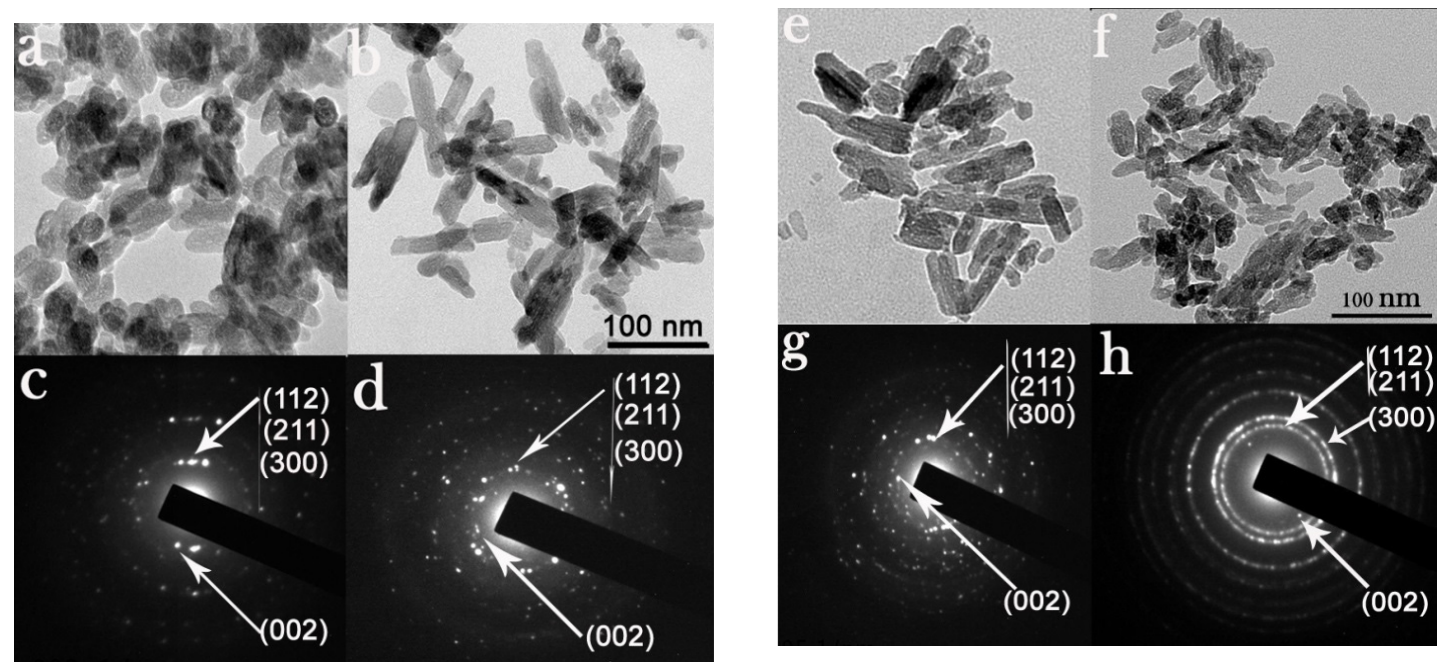

Fig.7. TEM of HA/CS composites with different ratios of different acids (a) HA/CS/2AA, (b) HA/CS/2Ac, (e) HA/ CS/4AA and (f) HA/CS/4Ac.

(c, $d, g$ and $h$ ) corresponded to diffraction image of the sample, respectively. 


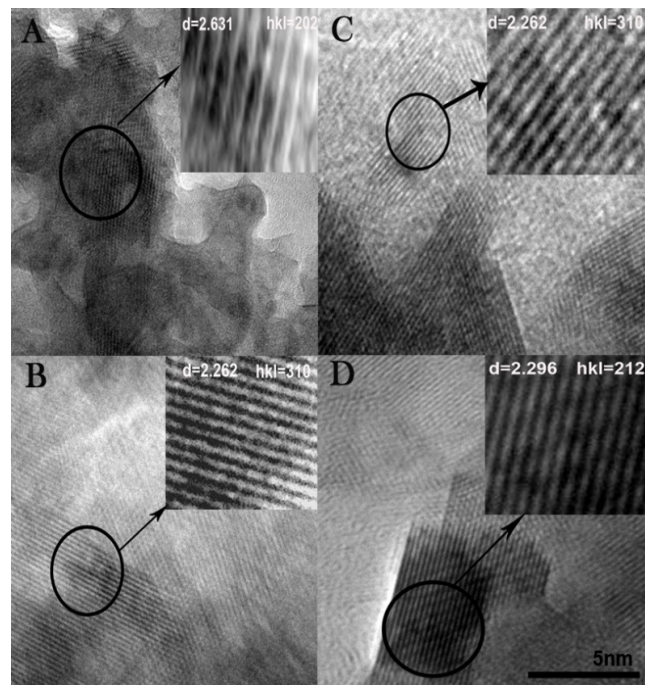

Fig. 8. High resolution-TEM of CS/HA nanocomposites A) HA/CS/2AA, B) HA/CS/4AA, C) HA/ $\mathrm{CS} / 2 \mathrm{Ac}$ and D) $\mathrm{HA} / \mathrm{CS} / \mathbf{4 A}$

interaction between HA and CS and its influence on the crystallinity and morphology of the in situ prepared HA. We found that using acrylic acid as a solvent reduced the interaction between HA and CS which resulted in larger HA particles. On the other hand, using acetic acid was found to have no influence on this interaction which leads to an inhibition in HA growth especially in direction of a-axis. The results in this article are important for designing an appropriate nanocomposite with good physical properties to be used as scaffold for tissue engineering purposes.

\section{References}

1. Hench, L.L., Biomaterials: a forecast for the future. Biomaterials, 19(16), p. 1419-1423 (1998).

2. Yuan, H. et al., Experimental study of natural hydroxyapatite/chitosan composite on reconstructing bone defects. Journal of Nanjing Medical University, 22(6), p. 372-375 (2008).

3. Betz, R.R., Limitations of autograft and allograft: new synthetic solutions. Orthopedics, 25 (5), p. S561-S570 (2002).

4. Salgado, A.J., Coutinho,O.P. and Reis, R.L., Bone tissue engineering: state of the art and future trends. Macromolecular Bioscience, 4 (8), p. 743-765 (2004).

5. Langer, R. and Tirrell, D.A., Designing materials for biology and medicine. Nature, 428 (6982), p. 487-492 (2004)..

6. Bhowmik, R., Katti, K.S. and Katti, D., Molecular dynamics simulation of hydroxyapatite-polyacrylic acid interfaces. Polymer, 48 (2), p. 664-674 (2007).

7. Sailaja, G. et al., Hydroxyapatite filled chitosanpolyacrylic acid polyelectrolyte complexes. Journal of Materials Science, 38 (17), p. 3653-3662 (2003).

8. Kumar, R. et al., Chitosan-mediated crystallization and assembly of hydroxyapatite nanoparticles into hybrid nanostructured films. Journal of the Royal Society Interface, 5 (21), p. 427-439 (2008).

9. Cai, X. et al., Preparation and characterization of homogeneous chitosan-polylactic acid/ hydroxyapatite nanocomposite for bone tissue engineering and evaluation of its mechanical properties. Acta Biomaterialia, 5 (7), p. 2693-2703 (2009).

10. Rogina, A., Ivanković, M. and Ivanković,H., Preparation and characterization of nanohydroxyapatite within chitosan matrix. Materials Science and Engineering, C, 33 (8), p. 4539-4544 (2013).

11. Kikuchi, M. et al., Self-organization mechanism in a bone-like hydroxyapatite/collagen nanocomposite synthesized in vitro and its biological reaction in vivo. Biomaterials, 22 (13), p. 1705-1711 (2001).

12. Lee, W.H. et al., High protein adsorptive capacity of amino acid-functionalized hydroxyapatite. Journal of Biomedical Materials Research, Part A, 101 (3), p. 873-883 (2013).

13. Zhang, X. et al., Catalytic dehydration of lactic acid to acrylic acid over modified ZSM-5 catalysts. Chemical Engineering Journal, 284, p. $934-941$ (2016).

14. Zhang, H. et al., Synthesis of hollow hybrid hydroxyapatite microspheres based on chitosanpoly (acrylic acid) microparticles. Biomedical Materials, 4 (3), p. 031002 (2009).

15. Hu, Y. et al., Synthesis and stimuli-responsive properties of chitosan/poly (acrylic acid) hollow nanospheres. Polymer, 46 (26), p. 12703-12710 (2005).

16. Yamaguchi, I. et al., Preparation and microstructure analysis of chitosan/hydroxyapatite nanocomposites. Journal of Biomedical Materials Research, 55 (1), p. 20-27 (2001).

17. Li, Z. et al., Preparation and in vitro investigation of chitosan/nano-hydroxyapatite composite used as bone substitute materials. Journal of Materials Science: Materials in Medicine, 16 (3), p. 213-219 (2005). 
18. Pillai, C., Paul, W. and Sharma, C.P., Chitin and chitosan polymers: Chemistry, solubility and fiber formation. Progress in Polymer Science, 34 (7), p. 641-678 (2009).

19. Shen, D. et al., Structure and properties of polyacrylic acid modified hydroxyapatite/liquid crystal polymer composite. Journal of Reinforced Plastics and Composites, 30 (13), p. 1155-1163 (2011).

20. Yao, J. et al., Hydroxyapatite nanostructure material derived using cationic surfactant as a template. Journal of Materials Chemistry, 13 (12), p. 30533057 (2003).

21. Larsson, M. et al., Biomedical applications and colloidal properties of amphiphilically modified chitosan hybrids. Progress in Polymer Science, 38 (9), p. 1307-1328 (2013).

22. Chang, M.C., Ko, C.-C. and Douglas, W.H. Preparation of hydroxyapatite-gelatin nanocomposite. Biomaterials, 24 (17), p. 2853-2862 (2003).

23. Fang, W. et al., Hydroxyapatite Crystal Formation in the Presence of Polysaccharide. Crystal Growth \& Design, 16(3),p. 1247-1255 (2016).

24. Maachou, H., et al., XPS analysis of chitosanhydroxyapatite biomaterials: from elements to compounds. Surface and Interface Analysis, 45(7), p. 1088-1097 (2013).

25. Liao, J.-D., Lin,S.-P. and Wu,Y.-T., Dual properties of the deacetylated sites in chitosan for molecular immobilization and biofunctional effects. Biomacromolecules, 6 (1), p. 392-399 (2005).

26. Chang, M.C. and Tanaka, J., XPS study for the microstructure development of hydroxyapatitecollagen nanocomposites cross-linked using glutaraldehyde. Biomaterials, 23 (18), p. 3879-3885 (2002).

27. Shen, J.-W. et al., Molecular simulation of protein adsorption and desorption on hydroxyapatite surfaces. Biomaterials, 29 (5), p. 513-532 (2008).

28. Wang, A. et al., Size-controlled synthesis of hydroxyapatite nanorods by chemical precipitation in the presence of organic modifiers. Materials Science and Engineering: C, 27 (4), p. 865-869 (2007).

29. Rusu, V.M. et al., Size-controlled hydroxyapatite nanoparticles as self-organized organic-inorganic composite materials. Biomaterials, 26 (26), p. 5414-5426 (2005).
30. Zhu, A. et al., Frabicating hydroxyapatite nanorods using a biomacromolecule template. Applied Surface Science, 257 (8), p. $3174-3179$ (2011).

31. Liou, S.-C., Chen, S.-Y. and Liu, D.-M., Synthesis and characterization of needlelike apatitic nanocomposite with controlled aspect ratios. Biomaterials, 24 (22), p. 3981-3988 (2003).

32. Di Chen, J. et al., Self-organization of hydroxyapatite nanorods through oriented attachment. Biomaterials, 28 (14), p. 2275-2280 (2007).

(Received: 15/6/2017; accepted: 13/7/2017) 
تأثبثير الأحماض المختلفة المتسخدة فى إذابة الكيتوزان على نمو حبيبات الهياروكسي

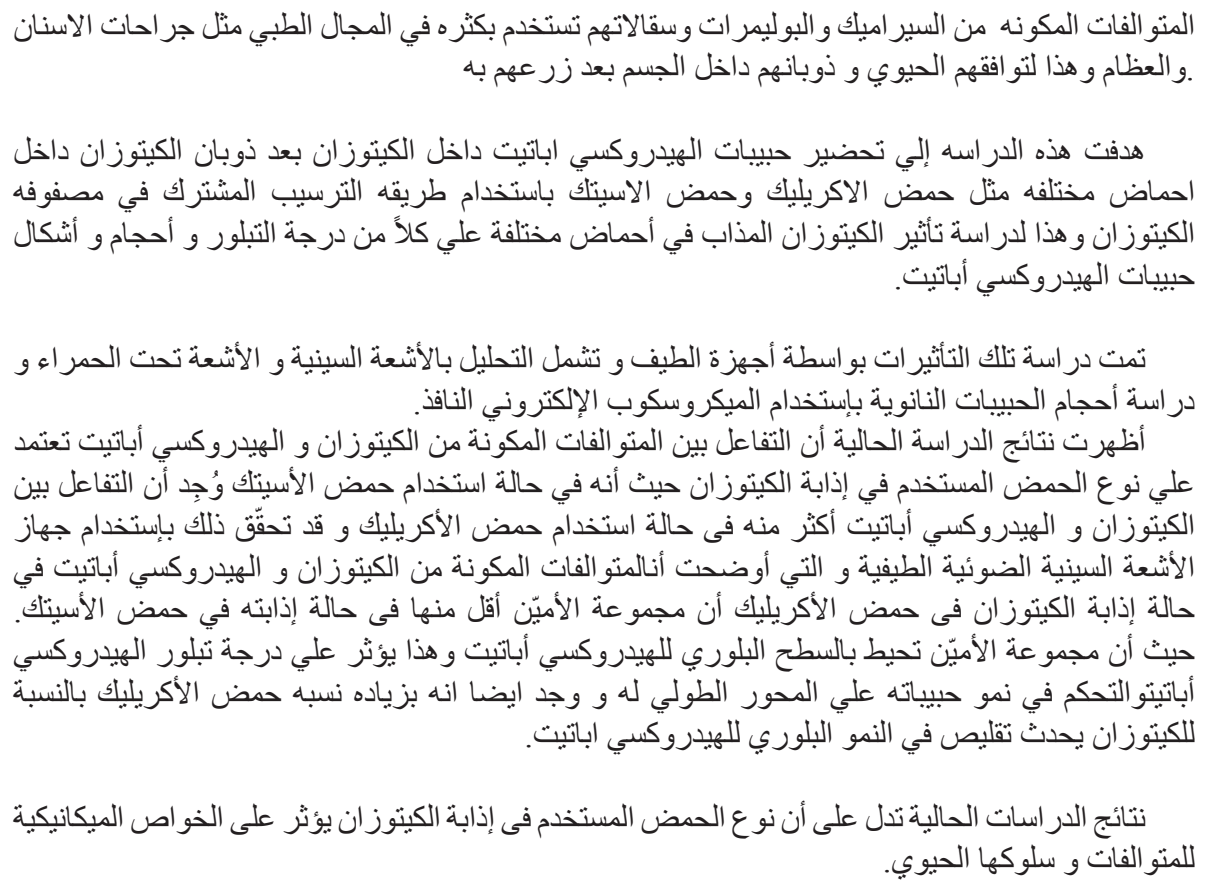

